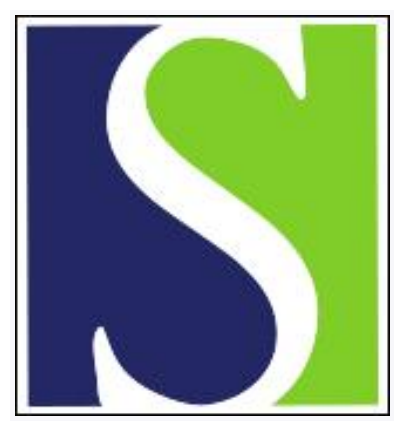

Scand J Work Environ Health 1976;2(2):57-70

https://doi.org/10.5271/sjweh.2817

Issue date: Jun 1976

Use of breath analysis to monitor methylene chloride exposure.

by Stewart RD, Hake CL, Wu A

Key terms: breath analysis; exposure; methylene chloride; methylene chloride exposure; occupational monitoring

This article in PubMed: www.ncbi.nlm.nih.gov/pubmed/959792

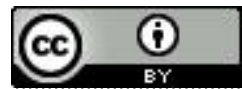




\title{
Use of breath analysis to monitor methylene chloride exposure
}

\author{
by RICHARD D. STEWART, M.D., M.P.H., F.A.C.P., CARL L. HAKE, Ph.D., \\ and ANTHONY WU, Ph.D. ${ }^{1}$
}

\begin{abstract}
STEWART, R.D., HAKE, C.L. and WU, A. Use of breath analysis to monitor methylene chloride exposure. Scand. $j$. work environ. \& health 2 (1976) 57-70. Twenty male and female subjects were exposed repetitively to methylene chloride $\left(\mathrm{CH}_{2} \mathrm{Cl}_{2}\right)$ vapor, $50,100,250$, and $500 \mathrm{ppm}$, for 1,3 , or $7.5 \mathrm{~h}$ in a controlled-environment chamber. Postexposure alveolar breath samples were collected in small glass breath tubes and analyzed for $\mathrm{CH}_{2} \mathrm{Cl}_{2}$ by gas chromatography. From these data a series of breath $\mathrm{CH}_{2} \mathrm{Cl}_{2}$ excretion curves were constructed that can be used to estimate the magnitude of a recent exposure. The $\mathrm{CH}_{2} \mathrm{CL}_{2}$ breath concentration in the immediate postexposure period accurately reflected the vapor concentration to which the subjects had been exposed most recently. Breath samples collected $1-2 \mathrm{~h}$ following exposure were accurate indicators of the time-weighted average vapor exposure experienced by the subjects during the previous $8 \mathrm{~h}$ of occupational exposure. Breath analysis offers a practical, noninvasive method for monitoring occupational exposure to $\mathrm{CH}_{2} \mathrm{Cl}_{2}$.
\end{abstract}

Key words: methylene chloride exposure, occupational monitoring, breath analysis.

To protect American workmen from excessive exposure to methylene chloride vapor (dichloromethane, $\mathrm{CH}_{2} \mathrm{Cl}_{2}$ ), a threshold limit value (TLV) of $200 \mathrm{ppm}$ is in effect (1). To insure compliance with this industrial standard, a reliable monitoring system is required. The usual method employed is the time-honored industrial hygiene survey which measures the concentration of methylene chloride in the workplace. Periodical industrial hygiene surveys are adequate in those industrial

1 Department of Environmental Medicine, The Medical College of Wisconsin and the Milwaukee County Medical Complex, Milwaukee, Wisconsin, U.S.A.

Reprint requests to: Dr. R. D. Stewart, AllenBradley Medical Science Laboratory, 8700 West Wisconsin Avenue, Milwaukee, Wisconsin 53226, U.S.A. settings in which the use of the solvent is predictable and unchanging. Work areas with variable and widely fluctuating methylene chloride concentrations ideally require continuous air monitoring, an expensive and frequently impractical procedure even for larger industries.

Inadvertent overexposure to methylene chloride poses one additional health hazard which reinforces the need to control workplace exposure. Once absorbed, this solvent is rapidly metabolized to carbon monoxide (CO) $(2,3,4,5,7,9,10,12)$, and excessive vapor exposure can result in the formation of toxic amounts of carboxyhemoglobin $(\mathrm{COHb})(9,10,12)$. The amount of $\mathrm{CO}$ formed from solvent metabolism is additive to that from other sources so that overexposure to $\mathrm{CO}$ is more likely in the indus- 
trial setting that already features appreciable $\mathrm{CO}$ exposure (12).

An alternative method of monitoring methylene chloride exposure has been advocated: the use of expired breath measurements to estimate occupational exposure similar to the use of breath analysis to estimate blood alcohol levels $(2,11,12)$. Because of the inherent economy and simplicity of breath analysis this investigation was undertaken to obtain the data required to estimate the magnitude of a methylene chloride exposure.

\section{EXPERIMENTAL PROCEDURE}

Twenty healthy adults of both sexes were exposed repetitively to known concentrations of methylene chloride vapor in a controlled-environment chamber $(12,14)$. These exposures were 1,3 , or $7.5 \mathrm{~h}$ in duration, were repetitive, and featured either nonfluctuating or widely fluctuating vapor concentrations.

\section{Exposure schedule}

The vapor concentrations and the duration of each exposure sequence are presented in tables 1 and 2 . The male subjects were exposed repetitively to methylene chloride vapor concentrations of 50, 100,

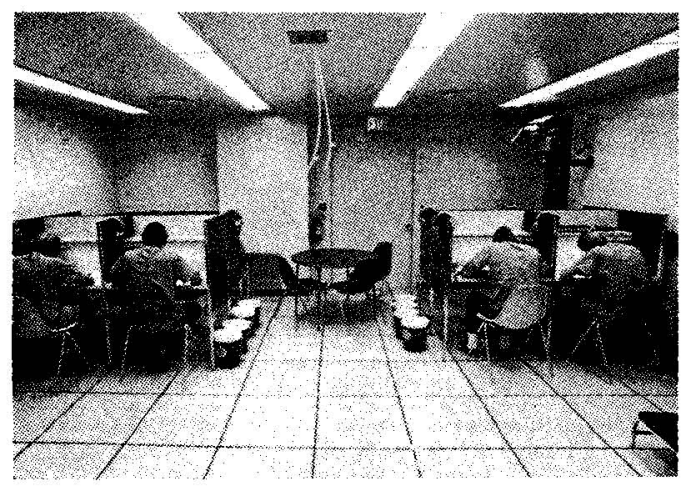

Fig. 1. Controlled-environment chamber, The Medical College of Wisconsin. The contaminated air enters through the four diffusers in the ceiling. Chamber air is monitored continuously by two independent analytical systems.
250 , or $500 \mathrm{ppm}$ for periods of 1,3 , and $7.5 \mathrm{~h}$ each day. During the second week of exposure to $250 \mathrm{ppm}$ the vapor concentration in the chamber was allowed to fluctuate from $188 \mathrm{ppm}$ to $313 \mathrm{ppm}$ (12). The female subjects were exposed to $250 \mathrm{ppm}$ for periods of 1,3 , or $7.5 \mathrm{~h}$ each day for 5 days.

\section{Exposure chamber}

All exposures were conducted in a controlled-environment chamber $6.1 \mathrm{~m} \times 6.1$ $\mathrm{m} \times 2.4 \mathrm{~m}$ in size (fig. 1). Details of the chamber and its operation have already been presented (14). Air flow through the chamber was approximately $42.5 \mathrm{~m}^{3} / \mathrm{min}$; $75 \%$ of the air was recirculated. Air temperature was maintained at 22.2$23.3^{\circ} \mathrm{C}$, while relative humidity ranged between $45-55 \%$. The methylene chloride was introduced by sweeping the concentrated vapor from a warm flask with a stream of air into the chamber's. circulating air. A reciprocal dual-piston pump maintained a steady flow of liquid methylene chloride into the flask.

\section{Analysis of exposure chamber atmosphere}

The methylene chloride (Fisher Scientific Co., Certified ACS, D-37) used in these studies was shown by infrared analysis to be $>99 \%$ pure.

The vapor concentration in the chamber atmosphere was recorded continuously by an infrared spectrometer equipped with a 20-min path-length gas cell which was flushed continuously with air drawn from the chamber through polyethylene tubing $6.4 \mathrm{~mm}$ in diameter. The absorbance of $13.3 \mu$ through a path-length of $2.25 \mathrm{~m}$ was measured. The infrared signal to the recorder was monitored each second by an on-line computer, which displayed the mean vapor concentration, as compared to standards, for each 30-s time interval of exposure and calculated the daily timeweighted-average exposures. Calibration standards of methylene chloride in purified air were prepared in saran bags and analyzed before, during, and after each daily exposure. A second infrared spectrometer was connected to an independent 

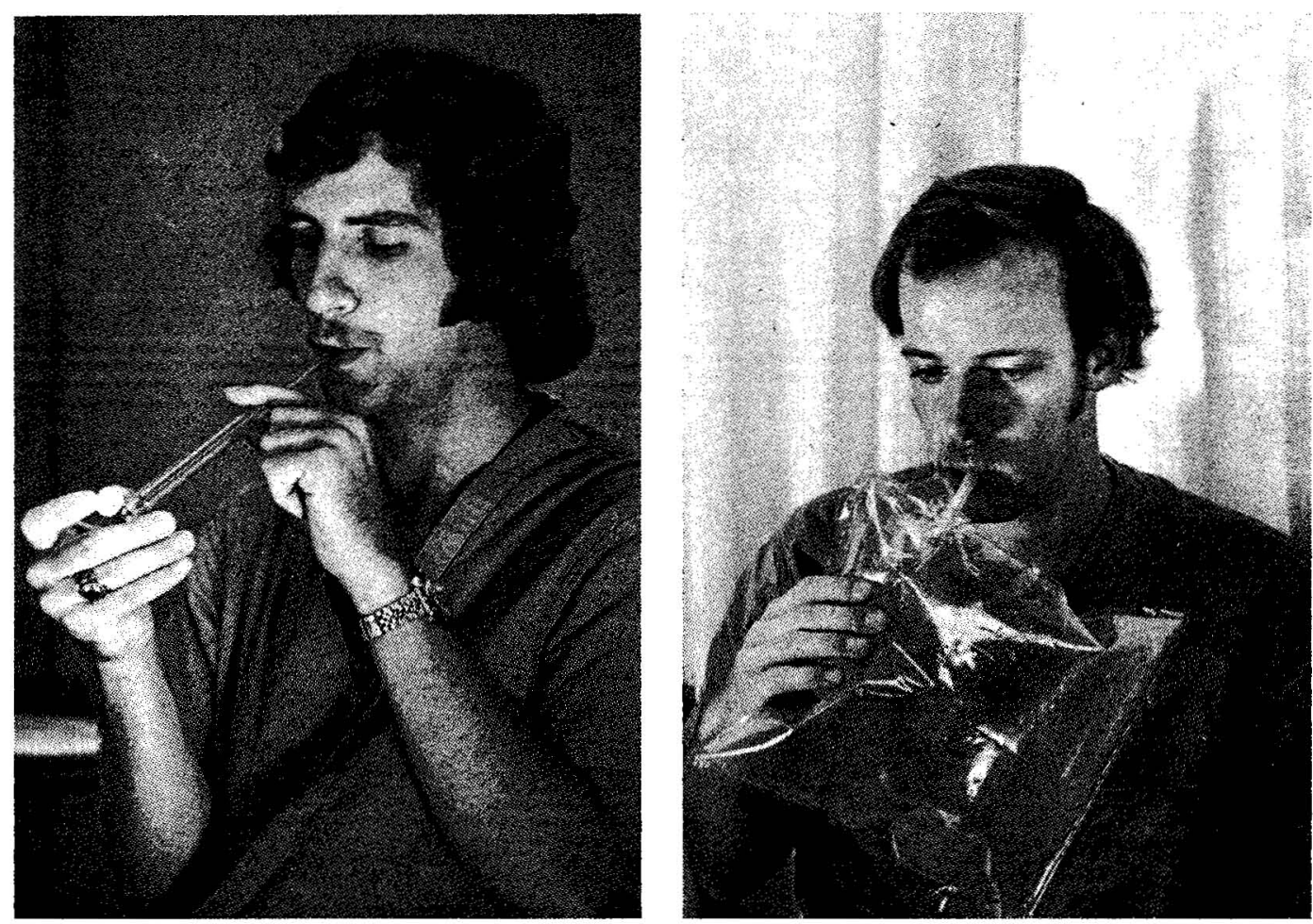

Fig. 2. The glass breath tube $\left(35 \mathrm{~cm}^{3}\right)$ has a predrilled hole in one cap for gas sampling. Both caps have saran liners that seal the glass chamber and permit storage of the breath sample for prolonged intervals (13). The saran bag $\left(5 \mathrm{dm}^{3}\right)$, an alternative for breath collection, provides enough sample for infrared analysis but permits only short-term breath storage (13).

polyethylene tubing loop, and it sampled the environmental chamber atmosphere in a manner similar to the first. This second instrument served as a back-up spectrometer and as an analytical check on the infrared spectrometer connected to the computer.

The second independent method of monitoring the chamber atmosphere during the study with female subjects was through the use of a gas chromatograph equipped with a flame ionization detector and an automatic sampling device. A sample of the chamber atmosphere was automatically swept into the carrier gas $\left(\mathrm{N}_{2}\right)$ of the chromatograph every $3 \mathrm{~min}$. A Varian Aerograph model 2700, Moduline ${ }^{\circledR}$ gas chromatograph was fitted with a stainless steel column, $35.6 \times 0.3 \mathrm{~cm}$, packed with Poropak Q, 60/80 mesh. The column was preconditioned at $200^{\circ} \mathrm{C}$ for $24 \mathrm{~h}$ prior to its use. Throughout the study, the column was baked at $150^{\circ} \mathrm{C}$ when it was not in use. The operating conditions of the gas chromatograph were the following: carrier gas flow rate $35 \mathrm{~cm}^{3} / \mathrm{min}$, column temperature $150^{\circ} \mathrm{C}$, injection port $230^{\circ} \mathrm{C}$, and detector $200^{\circ} \mathrm{C}$.

\section{Subjects}

The subjects were selected from the Caucasian, middle-class, working population of the Milwaukee metropolitan area. They were recruited for this study by a private employment agency. After the purpose of the study and the nature of the procedures to be used were fully explained to them, all subjects signed an informed consent form.

Eleven healthy males volunteered. Their ages ranged from 20 to 39 years, height from 170 to $190 \mathrm{~cm}$, and weight from 57 to $93.5 \mathrm{~kg}$. None were obese. 
The ages of the nine participating females ranged from 20 to 41 years; their height, from 158 to $169 \mathrm{~cm}$; and their weight, from 46.3 to $75.0 \mathrm{~kg}$. None were obese.
One male subject in each exposure group smoked cigarettes. All of the females and the remaining male subjects were nonsmokers. The smokers from the 7.5-h and 1-h groups smoked one-half to one pack

Table 1 a. Methylene chloride exposure schedule (male subjects).

\begin{tabular}{|c|c|c|c|c|c|c|c|c|}
\hline \multirow{3}{*}{ Week } & \multirow{3}{*}{$\begin{array}{c}\text { Day } \\
\text { of } \\
\text { week }\end{array}$} & \multirow{3}{*}{$\begin{array}{l}\text { Desired } \\
\text { concentration } \\
(\mathrm{ppm})\end{array}$} & \multicolumn{6}{|c|}{ Actual time-weighted average vapor concentration (ppm) } \\
\hline & & & \multicolumn{2}{|c|}{$7.5-\mathrm{h}$} & \multicolumn{2}{|c|}{$3-\mathrm{h}$} & \multicolumn{2}{|c|}{$1-\mathrm{h}$} \\
\hline & & & Mean & $\pm \mathrm{SD}$ & Mean & $\pm \mathrm{SD}$ & Mean & $\pm \mathrm{SD}$ \\
\hline 1 & $\begin{array}{l}4 \\
5\end{array}$ & $\begin{array}{l}0 \\
0\end{array}$ & $\begin{array}{l}0 \\
0\end{array}$ & & $\begin{array}{l}0 \\
0\end{array}$ & & $\begin{array}{l}0 \\
0\end{array}$ & \\
\hline 2 & $\begin{array}{l}1 \\
2 \\
3 \\
4 \\
5\end{array}$ & $\begin{array}{c}\mathbf{5 0} \\
\text { (minimal } \\
\text { fluctuation) }\end{array}$ & $\begin{array}{l}49.8 \\
48.7 \\
50.0 \\
50.0 \\
50.1\end{array}$ & $\begin{array}{l}2.3 \\
4.6 \\
2.6 \\
1.4 \\
2.1\end{array}$ & $\begin{array}{l}49.6 \\
50.2 \\
49.9 \\
50.1 \\
49.2\end{array}$ & $\begin{array}{l}2.4 \\
2.2 \\
1.6 \\
1.2 \\
1.9\end{array}$ & $\begin{array}{l}47.4 \\
47.1 \\
49.6 \\
49.9 \\
50.3\end{array}$ & $\begin{array}{l}3.0 \\
4.1 \\
2.2 \\
0.6 \\
2.3\end{array}$ \\
\hline 3 & $\begin{array}{l}1 \\
2 \\
3 \\
4 \\
5\end{array}$ & $\begin{array}{c}250 \\
\text { (minimal } \\
\text { fluctuation) }\end{array}$ & $\begin{array}{l}248.3 \\
249.9 \\
250.0 \\
250.0 \\
249.8\end{array}$ & $\begin{array}{r}15.5 \\
8.6 \\
9.4 \\
7.6 \\
9.5\end{array}$ & $\begin{array}{l}250.3 \\
251.4 \\
248.9 \\
249.9 \\
249.7\end{array}$ & $\begin{array}{r}9.0 \\
9.0 \\
12.1 \\
7.0 \\
7.8\end{array}$ & $\begin{array}{l}248.1 \\
251.7 \\
243.4 \\
251.8 \\
249.7\end{array}$ & $\begin{array}{r}10.0 \\
9.9 \\
3.1 \\
9.3 \\
4.8\end{array}$ \\
\hline 4 & $\begin{array}{l}1 \\
2 \\
3 \\
4 \\
5\end{array}$ & $\begin{array}{c}250 \\
\text { (wide } \\
\text { fluctuation) }\end{array}$ & $\begin{array}{l}249.2 \\
247.1 \\
250.1 \\
250.1 \\
250.1\end{array}$ & $\begin{array}{l}50.3 \\
45.3 \\
52.2 \\
51.0 \\
51.2\end{array}$ & $\begin{array}{l}249.2 \\
247.6 \\
250.9 \\
249.0 \\
250.3\end{array}$ & $\begin{array}{l}\mathbf{5 2 . 3} \\
\mathbf{5 0 . 7} \\
\mathbf{5 0 . 9} \\
51.7 \\
47.1\end{array}$ & $\begin{array}{l}248.7 \\
248.6 \\
254.2 \\
252.6 \\
247.8\end{array}$ & $\begin{array}{l}47.0 \\
45.6 \\
52.8 \\
48.1 \\
49.1\end{array}$ \\
\hline 5 & $\begin{array}{l}1 \\
2 \\
3 \\
4 \\
5\end{array}$ & $\begin{array}{c}100 \\
\text { (minimal } \\
\text { fluctuation) }\end{array}$ & $\begin{array}{r}99.7 \\
100.0 \\
100.1 \\
99.0 \\
100.5\end{array}$ & $\begin{array}{l}4.1 \\
4.2 \\
9.4 \\
5.1 \\
5.8\end{array}$ & $\begin{array}{r}99.4 \\
99.0 \\
100.4 \\
100.6 \\
100.2\end{array}$ & $\begin{array}{l}2.2 \\
3.2 \\
6.4 \\
4.1 \\
3.0\end{array}$ & $\begin{array}{r}101.9 \\
100.2 \\
103.4 \\
99.8 \\
101.2\end{array}$ & $\begin{array}{l}4.3 \\
2.6 \\
9.4 \\
1.5 \\
4.2\end{array}$ \\
\hline & $\begin{array}{l}1 \\
2\end{array}$ & 0 & $\begin{array}{l}0 \\
0\end{array}$ & & $\begin{array}{l}0 \\
0\end{array}$ & & $\begin{array}{l}0 \\
0\end{array}$ & \\
\hline 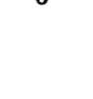 & $\begin{array}{l}3 \\
4\end{array}$ & $\begin{array}{c}500 \\
\text { (minimal } \\
\text { fluctuation) }\end{array}$ & $\begin{array}{l}499.5 \\
497.1\end{array}$ & $\begin{array}{l}15.9 \\
11.7\end{array}$ & $\begin{array}{l}494.2 \\
494.1\end{array}$ & $\begin{array}{r}10.7 \\
9.5\end{array}$ & $\begin{array}{l}495.3 \\
498.5\end{array}$ & $\begin{array}{r}30.1 \\
8.4\end{array}$ \\
\hline
\end{tabular}

Table $1 \mathrm{~b}$. Methylene chloride exposure schedule (female subjects).

\begin{tabular}{|c|c|c|c|c|c|c|c|c|}
\hline \multirow{3}{*}{ Week } & \multirow{3}{*}{$\begin{array}{c}\text { Day } \\
\text { of } \\
\text { week }\end{array}$} & \multirow{3}{*}{$\begin{array}{c}\text { Desired } \\
\text { concentration } \\
(\mathrm{ppm})\end{array}$} & \multicolumn{6}{|c|}{ Actual time-weighted average vapor concentration (ppm) } \\
\hline & & & \multicolumn{2}{|c|}{$7.5-\mathrm{h}$} & \multicolumn{2}{|c|}{$3-\mathrm{h}$} & \multicolumn{2}{|c|}{$1-\mathrm{h}$} \\
\hline & & & Mean & $\pm \mathrm{SD}$ & Mean & $\pm \mathrm{SD}$ & Mean & $\pm \mathrm{SD}$ \\
\hline 1 & 5 & 0 & 0 & & 0 & & 0 & \\
\hline 2 & $\begin{array}{l}1 \\
2 \\
3 \\
4 \\
5\end{array}$ & $\begin{array}{c}250 \\
\text { (minimal } \\
\text { fluctuation) }\end{array}$ & $\begin{array}{l}248.7 \\
249.7 \\
249.9 \\
250.1 \\
243.4\end{array}$ & $\begin{array}{l}7.7 \\
6.6 \\
5.9 \\
5.3 \\
6.4\end{array}$ & $\begin{array}{l}249.7 \\
249.5 \\
249.3 \\
251.3 \\
244.9\end{array}$ & $\begin{array}{l}8.5 \\
6.6 \\
4.1 \\
6.0 \\
7.3\end{array}$ & $\begin{array}{l}246.4 \\
250.1 \\
248.6 \\
248.6 \\
248.5\end{array}$ & $\begin{array}{r}11.1 \\
8.0 \\
2.9 \\
5.9 \\
3.9\end{array}$ \\
\hline 3 & 1 & 0 & 0 & & 0 & & 0 & \\
\hline
\end{tabular}


Table 2 a. Methylene chloride breath concentration of sedentary males exposed to 50 ppm.

\begin{tabular}{|c|c|c|c|c|c|c|c|}
\hline Time & Number & Mean & Range & Number & Mean & Range & $\mathrm{SD}$ \\
\hline & \multicolumn{3}{|c|}{ Single exposure for $1 \mathrm{~h}$} & \multicolumn{4}{|c|}{5 daily 1-h exposures } \\
\hline $1 \mathrm{~min}$, preexit & 3 & 11.6 & $11.2-12.0$ & 8 & 11.8 & $8.4-15.7$ & 2.22 \\
\hline 1 min, post & 2 & 5.5 & $5.3-5.7$ & 9 & 4.7 & $1.5-8.5$ & 2.06 \\
\hline $15 \mathrm{~min}$, post & $\overline{3}$ & 3.9 & $0.9-7.8$ & 10 & 2.6 & $0.9-7.8$ & 2.05 \\
\hline $30 \mathrm{~min}$, post & 3 & 1.8 & $1.7-2.1$ & 10 & 1.4 & $0.3-2.7$ & 0.76 \\
\hline $60 \mathrm{~min}$, post & 2 & 1.0 & $0.9-1.2$ & 8 & 0.6 & $0.2-1.2$ & 0.36 \\
\hline $120 \mathrm{~min}$, post & 2 & 0.4 & $0.3-0.5$ & 8 & 0.3 & $0.2-0.5$ & 0.13 \\
\hline \multirow[t]{2}{*}{$180 \mathrm{~min}$, post } & 2 & 1.2 & $1.1-1.2$ & 8 & 0.4 & $0.2-1.2$ & 0.45 \\
\hline & \multicolumn{3}{|c|}{ Single exposure for $3 \mathrm{~h}$} & \multicolumn{4}{|c|}{5 daily $3-\mathrm{h}$ exposures } \\
\hline $1 \mathrm{~min}$, preexit & 3 & 12.5 & $11.7-13.2$ & 12 & 13.8 & $11.3-18.1$ & 2.10 \\
\hline $1 \mathrm{~min}$, post & 3 & 7.4 & $5.2-9.4$ & 7 & 5.8 & $3.0-9.4$ & 2.24 \\
\hline $15 \mathrm{~min}$, post & 3 & 4.1 & $3.6-4.5$ & 8 & 4.0 & $2.4-5.0$ & 0.89 \\
\hline $30 \mathrm{~min}$, post & 3 & 2.2 & $1.7-2.4$ & 9 & 1.9 & $0.8-2.8$ & 0.72 \\
\hline $60 \mathrm{~min}$, post & 2 & 0.9 & $0.4-1.4$ & 8 & 1.1 & $0.4-2.1$ & 0.67 \\
\hline $120 \mathrm{~min}$, post & 3 & 1.0 & $0.2-2.1$ & 9 & 0.9 & $0.2-2.1$ & 0.57 \\
\hline \multirow[t]{2}{*}{$180 \mathrm{~min}$, post } & 2 & 1.3 & $0.7-1.8$ & 8 & 0.7 & $0.3-1.8$ & 0.46 \\
\hline & \multicolumn{3}{|c|}{ Single exposure for $7.5 \mathrm{~h}$} & \multicolumn{4}{|c|}{5 daily $7.5-\mathrm{h}$ exposures } \\
\hline $1 \mathrm{~min}$, preexit & 4 & 14.2 & $13.1-15.5$ & 16 & 15.0 & $12-22.6$ & 2.76 \\
\hline $1 \mathrm{~min}$, post & 4 & $\begin{array}{r}17.4 \\
8.4\end{array}$ & $5.0-11.3$ & 16 & 7.6 & $4.5-12.9$ & 2.16 \\
\hline $15 \mathrm{~min}$, post & 4 & 4.7 & $4.0-5.7$ & 16 & 5.2 & $3.2-8.1$ & 1.25 \\
\hline $30 \mathrm{~min}$, post & 4 & 3.6 & $2.8-5.0$ & 16 & 3.6 & $2.1-5.5$ & 0.97 \\
\hline $60 \mathrm{~min}$, post & 4 & 1.4 & $0.8-1.9$ & 12 & 1.8 & $0.8-2.4$ & 0.46 \\
\hline $120 \mathrm{~min}$, post & 4 & 0.9 & $0.4-1.5$ & 12 & 1.1 & $0.4-1.7$ & 0.37 \\
\hline 180 min, post & 3 & 0.9 & $0.6-1.1$ & 11 & 0.9 & $0.6-1.4$ & 0.21 \\
\hline
\end{tabular}

Table $2 \mathrm{~b}$. Methylene chloride breath concentration of sedentary males exposed to $100 \mathrm{ppm}$.

\begin{tabular}{|c|c|c|c|c|c|c|c|}
\hline \multirow[t]{2}{*}{ Time } & Number & Mean & Range & Number & Mean & Range & SD \\
\hline & \multicolumn{3}{|c|}{ Single exposure for $1 \mathrm{~h}$} & \multicolumn{4}{|c|}{5 daily $1-\mathrm{h}$ exposures } \\
\hline $1 \mathrm{~min}$, preexit & 3 & 35.6 & $28.0-41.9$ & 14 & 36.1 & $28-41.9$ & 4.56 \\
\hline 1 min, post & 3 & 17.6 & $13.6-21.3$ & 12 & 19.1 & $10.5-29.5$ & 6.16 \\
\hline 15 min, post & 2 & 6.9 & $4.9-8.9$ & 12 & 7.9 & $3.4-12.8$ & 2.43 \\
\hline $30 \mathrm{~min}$, post & 3 & 3.7 & $3.2-4.1$ & 15 & 5.0 & $1.7-9.0$ & 2.14 \\
\hline $60 \mathrm{~min}$, post & 3 & 2.0 & $1.5-2.4$ & 13 & 3.0 & $1.4-5.1$ & 1.23 \\
\hline $120 \mathrm{~min}$, post & 3 & 0.9 & $0.3-1.2$ & 15 & 0.9 & $0.3 \longrightarrow 1.7$ & 0.40 \\
\hline \multirow[t]{2}{*}{180 min, post } & 3 & 0.8 & $0.7-0.9$ & 13 & 0.7 & $0.2-1.6$ & 0.36 \\
\hline & Singl & expos & for $3 \mathrm{~h}$ & \multicolumn{4}{|c|}{5 daily $3-\mathrm{h}$ exposures } \\
\hline $1 \mathrm{~min}$, preexit & 3 & 34.1 & $26.2-41.8$ & 12 & 36.9 & $25.3-44.5$ & 5.99 \\
\hline $1 \mathrm{~min}$, post & 3 & 19.7 & $18.2-21.1$ & 14 & 19.4 & $11.3-29.2$ & 4.70 \\
\hline $15 \mathrm{~min}$, post & 3 & 10.0 & $9.6-10.5$ & 15 & 9.9 & $7.4-14.6$ & 2.00 \\
\hline $30 \mathrm{~min}$, post & 3 & 5.0 & $2.3-6.7$ & 15 & 6.1 & $2.3-9.4$ & 1.60 \\
\hline $60 \mathrm{~min}$, post & 3 & 3.7 & $3.5-3.9$ & 15 & 3.8 & $2.4-5.9$ & 0.90 \\
\hline $120 \mathrm{~min}$, post & 3 & 1.6 & $0.9-2.5$ & 14 & 1.9 & $0.9-3.0$ & 0.61 \\
\hline \multirow[t]{2}{*}{$180 \mathrm{~min}$, post } & 1 & 0.9 & & 8 & 0.8 & $0.5-2$ & 0.55 \\
\hline & \multicolumn{3}{|c|}{ Single exposure for $7.5 \mathrm{~h}$} & \multicolumn{4}{|c|}{5 daily 7.5-h exposures } \\
\hline $1 \mathrm{~min}$, preexit & 4 & 32.9 & $26.6-39.9$ & 20 & 33.4 & $17.5-49.3$ & 8.27 \\
\hline $1 \mathrm{~min}$, post & 4 & 20.3 & $18.6-23.4$ & 20 & 21.9 & $12.3-32.3$ & 5.22 \\
\hline $15 \mathrm{~min}$, post & 4 & 9.5 & $8.4-10.5$ & 19 & 11.6 & $8.4-17.8$ & 2.54 \\
\hline $30 \mathrm{~min}$, post & 4 & 6.1 & $4.7-7.5$ & 20 & 7.9 & $4.7-13.3$ & 2.36 \\
\hline $60 \mathrm{~min}$, post & 4 & 4.0 & $3.1-5.2$ & 20 & 4.5 & $2.8-6.7$ & 4.45 \\
\hline $120 \mathrm{~min}$, post & 4 & 3.7 & $1.6-5.2$ & 18 & 3.2 & $1.6-5.2$ & 1.12 \\
\hline $180 \mathrm{~min}$, post & 4 & 2.4 & $0.9-3.9$ & 16 & 2.1 & $0.4-3.9$ & 1.05 \\
\hline
\end{tabular}


Table 2c. Methylene chloride breath concentration of sedentary males exposed to $250 \mathrm{ppm}$.

\begin{tabular}{|c|c|c|c|c|c|c|c|}
\hline Time & Number & Mean & Range & Number & Mean & Range & $\mathrm{SD}$ \\
\hline & \multicolumn{3}{|c|}{ Single exposure for $1 \mathrm{~h}$} & \multicolumn{4}{|c|}{5 daily $1-\mathrm{h}$ exposures } \\
\hline $1 \mathrm{~min}$, preexit & 3 & 74.7 & $63.6-88.6$ & 15 & 84.3 & $63.6-103.2$ & 11.9 \\
\hline $1 \mathrm{~min}$, post & - & - & - & 7 & 43.0 & $36.7-51.6$ & 4.5 \\
\hline $15 \mathrm{~min}$, post & - & - & - & 9 & 21.6 & $15.9-31.6$ & 4.6 \\
\hline $30 \mathrm{~min}$, post & 1 & 11.9 & 一 & 8 & 14.2 & $11.4-19.4$ & 3.2 \\
\hline $60 \mathrm{~min}$, post & 2 & 6.8 & $6.1-7.5$ & 10 & 7.1 & $4.8-9.2$ & 1.5 \\
\hline $120 \mathrm{~min}$, post & 2 & 1.5 & $1.4-1.6$ & 13 & 2.1 & $1.4-3.0$ & 0.6 \\
\hline $180 \mathrm{~min}$, post & 3 & 0.9 & $0.8-1.2$ & 14 & 1.3 & $0.8-2.5$ & 0.5 \\
\hline \multirow[t]{2}{*}{$1,350 \mathrm{~min}$, post } & 一 & - & - & 4 & 0.2 & $0.2-0.7$ & 0.2 \\
\hline & \multicolumn{3}{|c|}{ Single exposure for $3 \mathrm{~h}$} & \multicolumn{4}{|c|}{5 daily 3-h exposures } \\
\hline $1 \mathrm{~min}$, preexit & 3 & 86.0 & $79.4-95.8$ & 15 & 103.0 & $79.3-130.0$ & 14.4 \\
\hline $1 \mathrm{~min}$, post & - & - & - & 10 & 65.7 & $49.5-92.8$ & 14.2 \\
\hline $15 \mathrm{~min}$, post & 1 & $\Sigma 6.0$ & - & 11 & 35.8 & $23.4-52.9$ & 9.6 \\
\hline $30 \mathrm{~min}$, post & 2 & 16.4 & $16.3-16.5$ & 13 & 22.3 & $14.0-38.0$ & 6.9 \\
\hline $60 \mathrm{~min}$, post & 1 & 9.4 & - & 12 & 10.8 & $4.7-16.2$ & 3.8 \\
\hline $120 \mathrm{~min}$, post & 3 & 3.8 & $2.4-\quad 4.7$ & 13 & 4.8 & $2.4-9.3$ & 2.1 \\
\hline $180 \mathrm{~min}$, post & 3 & 2.1 & $1.7-2.7$ & 10 & 2.9 & $0.9-5.3$ & 1.4 \\
\hline \multirow[t]{2}{*}{$1,170 \mathrm{~min}$, post } & 2 & 0.4 & $0.3-0.6$ & 10 & 0.5 & $0.2-0.7$ & 0.2 \\
\hline & \multicolumn{3}{|c|}{ Single exposure for $7.5 \mathrm{~h}$} & \multicolumn{4}{|c|}{5 daily $7.5-\mathrm{h}$ exposures } \\
\hline $1 \mathrm{~min}$, preexit & 3 & 112.1 & $101.0-133.2$ & 16 & 119.3 & $69.6-160.2$ & 21.1 \\
\hline $1 \mathrm{~min}$, post & 3 & 69.1 & $52.8-84.6$ & 16 & 87.8 & $66.0-110.1$ & 16.0 \\
\hline $15 \mathrm{~min}$, post & 4 & 37.3 & $30.2-49.7$ & 18 & 47.2 & $30.2-64.9$ & 11.0 \\
\hline $30 \mathrm{~min}$, post & 3 & 33.0 & $26.2-43.1$ & 19 & 32.3 & $18.6-44.0$ & 7.0 \\
\hline $60 \mathrm{~min}$, post & 2 & 14.9 & $14.1-15.7$ & 16 & 19.5 & $11.5-27.9$ & 4.7 \\
\hline $120 \mathrm{~min}$, post & 2 & 5.8 & $4.5-7.1$ & 17 & 8.8 & $4.5-14.7$ & 2.9 \\
\hline $180 \mathrm{~min}$, post & 3 & 5.4 & $4.6-6.4$ & 16 & 6.7 & $3.8-16.0$ & 1.9 \\
\hline $960 \mathrm{~min}$, post & 3 & 0.4 & $0.3-0.5$ & 15 & 0.7 & $0.2-1.5$ & 0.4 \\
\hline $3,840 \mathrm{~min}$, post & 3 & 0.2 & $0.2-0.3$ & - & - & - & - \\
\hline
\end{tabular}

of cigarettes per day, while the smoker from the 3-h group smoked approximately two packs of cigarettes per day. Subjects who were smokers were not allowed to smoke during their stay in the controlledenvironment chamber.

All subjects were cautioned to abstain from the use of drugs and to limit their use of alcohol to very moderate amounts. Most of the subjects had no other wage-earning job during the time of the study, and none experienced any exposure to methylene chloride outside of the laboratory.

Each subject was given comprehensive medical examinations prior to inclusion in the study and during and following each exposure for the documentation of physical health status (12). Each had a normal pulmonary function as assessed by a forced maximum expiratory maneuver and a single breath CO diffusion measurement (12). Each had a normal cardiac function as assessed by the history and physical examination, the standard resting 12-lead ECG, continuous monitoring of lead II of the ECG by telemetry during expcsure, and a normal submaximal ECG stress test (12). Each had normal complete blood counts and clinical laboratory values (12).

The subjects were sedentary during each exposure except for the $6 \mathrm{~min}$ of exercise on the bicycle ergometer. The average minute respiratory volumes for the group while in the controlled-environment chamber ranged from $7-8 \mathrm{dm}^{3} / \mathrm{min}$. The medical surveillance program and all physiological testing procedures have been detailed previously (12)

\section{Breath sample collection and analysis}

Alveolar breath samples were obtained daily from each subject prior to entry into the environmental chamber, imme- 
diately prior to exiting from the chamber, and at the following times after exiting from the chamber (postexposure): 1, 15, $30 \mathrm{~min} ; 1,2,3 \mathrm{~h}$ (fig. 2). The last two samples were collected in duplicate. A preexposure sample the next day completed the sequence. Breath samples, with the exception noted later, were collected in $35-\mathrm{cm}^{3}$ glass tubes fitted at each end with screw caps containing saran liners $(8,13)$. One of the caps had a predrilled hole sealed with a saran disc through which an aliquot of the alveolar breath samples could be withdrawn. The subjects flushed the tube twice, then after holding their third breath for $30 \mathrm{~s}$, exhaled, and capped the tube while finishing the exhalation. For the collection of the alveolar breath sample just prior to exit from the environmental chamber the subject breathed through a polyethylene tube which extended through a porthole in the chamber door to a saran bag. Sampling of the saran bag was accomplished by puncturing its wall with a syringe needle.

Fifteen minutes after the end of the exposure on the fifth day of weeks $2,3,4$, and 5, a duplicate alveolar breath sample was collected from each subject, this one in a 4-1 saran bag. This breath sample was slow-scanned in a Beckman ${ }^{\circledR}$ IR-10 infrared spectrometer with a 10-m gas cell (13).

A Varian Aerograph model 2700 gas chromatograph equipped with a hydrogen flame ionization detector was used to determine methylene chloride in the breath samples. The gas chromatograph was fitted with two stainless steel columns (91.4 $\times$ $0.3 \mathrm{~cm}$ and $30.5 \times 0.3 \mathrm{~cm}$ ) each packed with Poropak Q, 50/80 mesh (Waters Associates, Inc.). The columns were preconditioned at $200^{\circ} \mathrm{C}$ overnight prior to use. The oper-

Table $2 d$. Methylene chloride breath concentration of sedentary males exposed to 250 ppm, widely fluctuating.

\begin{tabular}{|c|c|c|c|c|c|c|c|}
\hline Time & Number & Mean & Range & Number & Mean & Range & SD \\
\hline & \multicolumn{3}{|c|}{ Single exposure for $1 \mathrm{~h}$} & \multicolumn{4}{|c|}{5 daily $1-\mathrm{h}$ exposures } \\
\hline $1 \mathrm{~min}$, preexit & 3 & 67.5 & $61.7-73.5$ & 15 & 77.7 & $61.7-102.8$ & 12.8 \\
\hline $1 \mathrm{~min}$, post & 2 & 44.5 & $40.0-49.1$ & 10 & 50.2 & $40.0-61.9$ & 8.0 \\
\hline $15 \mathrm{~min}$, post & 2 & 16.3 & $14.7-17.9$ & 10 & 20.7 & $12.5-32.3$ & 6.3 \\
\hline $30 \mathrm{~min}$, post & - & - & - & 11 & 12.2 & $6.1-15.6$ & 2.6 \\
\hline $60 \mathrm{~min}$, post & 1 & 5.0 & - & 12 & 6.8 & $4.2-12.9$ & 2.4 \\
\hline $120 \mathrm{~min}$, post & 3 & 1.8 & $1.6-2.0$ & 15 & 2.5 & $1.2-4.7$ & 0.9 \\
\hline $180 \mathrm{~min}$, post & 3 & 1.3 & $1.0-1.5$ & 12 & 1.2 & $0.4-2.0$ & 0.4 \\
\hline \multirow[t]{2}{*}{$1,350 \mathrm{~min}$, post } & 3 & 0.2 & $0.1-0.3$ & 12 & 0.2 & $0.1-0.5$ & 0.1 \\
\hline & \multicolumn{3}{|c|}{ Single exposure for $3 \mathrm{~h}$} & \multicolumn{4}{|c|}{5 daily 3-h exposures } \\
\hline $1 \mathrm{~min}$, preexit & 2 & 85.0 & $78.9-91.4$ & 14 & 95.8 & $71.8-114.1$ & 12.8 \\
\hline $1 \mathrm{~min}$, post & 3 & 48.3 & $39.4-61.5$ & 14 & 60.0 & $39.4-76.2$ & 12.8 \\
\hline $15 \mathrm{~min}$, post & 2 & 29.6 & $28.8-30.3$ & 14 & 33.4 & $18.7-46.9$ & 8.3 \\
\hline $30 \mathrm{~min}$, post & 3 & 13.4 & $10.9-16.7$ & 15 & 20.4 & $10.9-31.2$ & 6.5 \\
\hline $60 \mathrm{~min}$, post & 3 & 9.2 & $6.8-10.9$ & 14 & 11.6 & $6.8-21.6$ & 3.9 \\
\hline $120 \mathrm{~min}$, post & 2 & 4.6 & $4.4-4.7$ & 12 & 4.9 & $3.0-7.0$ & 1.0 \\
\hline $180 \mathrm{~min}$, post & 3 & 2.5 & $0.8-3.6$ & 12 & 2.5 & $0.8-4.6$ & 1.1 \\
\hline \multirow[t]{2}{*}{$1,170 \mathrm{~min}$, post } & 3 & 0.5 & $0.4-0.6$ & 12 & 0.4 & $0.2-0.7$ & 0.2 \\
\hline & \multicolumn{3}{|c|}{ Single exposure for $7.5 \mathrm{~h}$} & \multicolumn{4}{|c|}{5 daily $7.5-h$ exposures } \\
\hline $1 \mathrm{~min}$, preexit & 4 & 91.4 & $71.4-109.4$ & 19 & 108.3 & $71.4-136.4$ & 14.9 \\
\hline $1 \mathrm{~min}$, post & 4 & 71.7 & $67.6-80.6$ & 16 & 77.3 & $65.7-96.1$ & 9.5 \\
\hline $15 \mathrm{~min}$, post & 4 & 30.2 & $28.2-32.9$ & 19 & 40.2 & $28.2-69.2$ & 9.5 \\
\hline $30 \mathrm{~min}$, post & 4 & 21.8 & $21.2-22.5$ & 18 & 24.2 & $18.4-32.7$ & 3.8 \\
\hline $60 \mathrm{~min}$, post & 2 & 12.7 & $12.4-13.0$ & 14 & 16.9 & $12.2-26.5$ & 4.4 \\
\hline $120 \mathrm{~min}$, post & 3 & 8.9 & $4.4-11.9$ & 18 & 9.2 & $4.4-12.2$ & 2.2 \\
\hline $180 \mathrm{~min}$, post & 3 & 7.2 & $3.7-9.2$ & 18 & 5.6 & $2.5-9.4$ & 2.0 \\
\hline $960 \mathrm{~min}$, post & 4 & 0.8 & $0.4-\quad 1.3$ & 16 & 1.1 & $0.3-\quad 2.8$ & 0.6 \\
\hline
\end{tabular}


ating conditions of the gas chromatograph were as follows: carrier gas (helium) flow rates, 10 and $20 \mathrm{ml} / \mathrm{min}$; column temperature, $75^{\circ} \mathrm{C}$; injection port, $150^{\circ} \mathrm{C}$; and detector, $200^{\circ} \mathrm{C}$. Standards at several concentrations to bracket the unknown levels were prepared with purified air as the diluent. One-cubic-centimeter aliquots were injected, and the concentration of the methylene chloride in the breath samples was obtained by direct comparison of peak heights to the standards.

\section{RESULTS}

Analysis of exposure chamber atmosphere

The daily time-weighted average concentrations of methylene chloride vapor in the chamber atmosphere, as determined by continuous infrared spectroscopy, are listed in table 1. Actual mean exposure concentrations were usually within $1 \%$ of planned concentrations.

\section{Medical surveillance}

The health of all subjects, with the exception of minor head colds and an occasional sore throat, remained excellent throughout the study. The only abnormal laboratory values reported which could be correlated in degree of abnormality with the magnitude of exposure to methylene chloride were venous blood $\mathrm{COHb}$ (12). Pulmonary and cardiac function remained normal and unchanged during the exposure.

\section{Breath analysis}

Methylene chloride and $\mathrm{CO}$ were present in the expired breath of each of the sub-

Table 2 e. Methylene chloride breath concentration of sedentary males and females exposed to $250 \mathrm{ppm}$.

\begin{tabular}{|c|c|c|c|c|}
\hline Time & Number & Mean & Range & $\mathrm{SD}$ \\
\hline & \multicolumn{4}{|c|}{5 daily $1-\mathrm{h}$ exposures } \\
\hline $1 \mathrm{~min}$, preexit & 43 & 83.5 & $61.7-108.2$ & 12.9 \\
\hline $1 \mathrm{~min}$, post & 32 & 53.9 & $36.7-78.8$ & 8.8 \\
\hline 15 min, post & 34 & 24.3 & $12.5-32.3$ & 4.4 \\
\hline $30 \mathrm{~min}$, post & 34 & 15.3 & $6.1-26.9$ & 3.0 \\
\hline $60 \mathrm{~min}$, post & 37 & 8.7 & $4.2-15.2$ & 2.0 \\
\hline 120 min, post & 41 & 2.6 & $1.2-5.4$ & 1.0 \\
\hline $180 \mathrm{~min}$, post & 38 & 1.7 & $0.4-3.6$ & 0.6 \\
\hline \multirow[t]{2}{*}{ 1,350 $\mathrm{min}$, post } & 31 & 1.0 & $0.2-\quad 4.0$ & 0.6 \\
\hline & \multicolumn{4}{|c|}{5 daily $3-\mathrm{h}$ exposures } \\
\hline $1 \mathrm{~min}$, preexit & 38 & 96.3 & $71.8-130.0$ & 12.9 \\
\hline $1 \mathrm{~min}$, post & 33 & 63.5 & $39.4-92.8$ & 12.9 \\
\hline $15 \mathrm{~min}$, post & 35 & 33.1 & $18.7-52.9$ & 8.5 \\
\hline $30 \mathrm{~min}$, post & 36 & 20.8 & $10.9-38.0$ & 6.6 \\
\hline $60 \mathrm{~min}$, post & 35 & 12.1 & $4.7-21.7$ & 3.9 \\
\hline 120 min, post & 34 & 4.5 & $1.2-9.3$ & 1.7 \\
\hline $180 \mathrm{~min}$, post & 30 & 2.7 & $0.8-5.3$ & 1.2 \\
\hline \multirow[t]{2}{*}{$1,170 \mathrm{~min}$, post } & 32 & 0.9 & $0.2-4.0$ & 0.6 \\
\hline & \multicolumn{4}{|c|}{5 daily $7.5-h$ exposures } \\
\hline $1 \mathrm{~min}$, preexit & 54 & 111.5 & $69.6-160.2$ & 18.1 \\
\hline $1 \mathrm{~min}$, post & 52 & 83.0 & $65.7--119.6$ & 14.5 \\
\hline 15 min, post & 56 & 42.6 & $28.2-69.2$ & 10.6 \\
\hline $30 \mathrm{~min}$, post & 57 & 30.1 & $18.4-55.8$ & 7.6 \\
\hline $60 \mathrm{~min}$, post & 50 & 20.3 & $11.5-37.7$ & 5.3 \\
\hline $120 \mathrm{~min}$, post & 53 & 8.3 & $4.4-14.7$ & 2.7 \\
\hline $180 \mathrm{~min}$, post & 53 & 5.7 & $2.5-16.0$ & 2.0 \\
\hline $960 \mathrm{~min}$, post & 51 & 1.4 & $0.2-4.8$ & 0.8 \\
\hline
\end{tabular}


Table $2 f$. Methylene chloride breath concentration of sedentary females exposed to $250 \mathrm{ppm}$.

\begin{tabular}{|c|c|c|c|c|c|c|c|}
\hline Time & Number & Mean & Range & Number & Mean & Range & SD \\
\hline & \multicolumn{3}{|c|}{ Single exposure for $1 \mathrm{~h}$} & \multicolumn{4}{|c|}{5 daily $1-\mathrm{h}$ exposures } \\
\hline $1 \mathrm{~min}$, preexit & 3 & 82.3 & $68.7-98.6$ & 13 & 89.3 & $68.7-108.2$ & 14.11 \\
\hline $1 \mathrm{~min}$, post & 3 & 56.7 & $51.0-62.6$ & 15 & 61.5 & $42.8-78.8$ & 10.62 \\
\hline $15 \mathrm{~min}$, post & 3 & 28.6 & $26.5-29.9$ & 15 & 28.3 & $23.9-31.9$ & 2.19 \\
\hline $30 \mathrm{~min}$, post & 3 & 17.9 & $17.0-19.1$ & 15 & 18.1 & $12.5-26.9$ & 3.19 \\
\hline $60 \mathrm{~min}$, post & 3 & 10.7 & $8.8-12.9$ & 15 & 11.2 & $8.6-15.2$ & 1.83 \\
\hline $120 \mathrm{~min}$, post & 3 & 2.8 & $1.6-3.5$ & 13 & 3.3 & $1.4-5.4$ & 1.27 \\
\hline $180 \mathrm{~min}$, post & 2 & 2.6 & $2.5-\quad 2.7$ & 12 & 2.7 & $1.2-3.6$ & 0.68 \\
\hline \multirow{2}{*}{$1,350 \mathrm{~min}$, post } & 3 & 2.7 & $1.3-4.0$ & 15 & 1.9 & $0.7-4.0$ & 0.90 \\
\hline & \multicolumn{3}{|c|}{ Single exposure for $3 \mathrm{~h}$} & \multicolumn{4}{|c|}{5 daily 3-h exposures } \\
\hline $1 \mathrm{~min}$, preexit & 2 & 81.3 & $75.5-87.1$ & 9 & 85.7 & $72.4-102.9$ & 10.14 \\
\hline $1 \mathrm{~min}$, post & 2 & 57.8 & $51.7-64.0$ & 9 & 66.5 & $51.7-89.1$ & 11.32 \\
\hline 15 min, post & 2 & 24.2 & $22.5-25.9$ & 10 & 29.7 & $22.5-44.9$ & 7.35 \\
\hline 30 min, post & 2 & 17.4 & $12.9-21.8$ & 8 & 19.1 & $12.9-32.6$ & 6.49 \\
\hline $60 \mathrm{~min}$, post & 2 & 13.8 & $7.0-21.7$ & 9 & 14.8 & $10.2-21.7$ & 3.87 \\
\hline $120 \mathrm{~min}$, post & 1 & 1.2 & & 9 & 3.6 & $1.2-7.0$ & 1.83 \\
\hline 180 min, post & 1 & 1.8 & & 8 & 2.7 & $1.6-\quad 3.9$ & 0.88 \\
\hline \multirow[t]{2}{*}{$1,170 \mathrm{~min}$, post } & 2 & 1.5 & $1.30-1.6$ & 10 & 1.8 & $0.6-4.0$ & 0.97 \\
\hline & \multicolumn{3}{|c|}{ Single exposure for $7.5 \mathrm{~h}$} & \multicolumn{2}{|c|}{5} & 7.5-h exposures & \\
\hline $1 \mathrm{~min}$, preexit & 4 & 116.7 & $100.0-136.3$ & 19 & 108.1 & $77.6-141.3$ & 18.33 \\
\hline $1 \mathrm{~min}$, post & 4 & 81.9 & $69.9-99.3$ & 20 & 83.7 & $55.3-119.6$ & 16.37 \\
\hline $15 \mathrm{~min}$, post & 4 & 40.1 & $24.7-50.7$ & 19 & 40.5 & $18.6-60.1$ & 11.31 \\
\hline $30 \mathrm{~min}$, post & 4 & 32.2 & $22.6-46.6$ & 20 & 33.3 & $19.7-55.8$ & 10.18 \\
\hline $60 \mathrm{~min}$, post & 4 & 22.1 & $13.7-30.1$ & 20 & 23.2 & $13.7-37.7$ & 6.14 \\
\hline $120 \mathrm{~min}$, post & 4 & 7.0 & $3.0-11.2$ & 18 & 6.9 & $3.0-13.6$ & 2.98 \\
\hline $180 \mathrm{~min}$, post & 3 & 4.5 & $2.1-6.7$ & 19 & 4.9 & $1.5-9.9$ & 2.10 \\
\hline $960 \mathrm{~min}$, post & 4 & 3.4 & $2.0-4.8$ & 20 & 2.3 & $0.8-4.8$ & 1.15 \\
\hline
\end{tabular}

jects after exposure to the four vapor concentrations. The postexposure methylene chloride breath data for male and female subjects are listed in table 2 . Examination of these breath data reveals that a predictable excretion pattern exists for each of the vapor concentrations studied. Several of the breath decay curves obtained in this study are graphically presented in figs. $3-5$.

A "family" of postexposure breath decay curves can be constructed from the methylene chloride breath data. Such a "family" is presented in fig. 3.

The concentration of methylene chloride in the breath after exposure is directly related to the time-weighted average concentration during exposure, the duration of exposure being constant. There was little difference between the mean breath concentration of those subjected to a nonfluctuating or widely fluctuating 250 -ppm vapor exposure (fig. 4). The variation in breath concentration between male subjects identically exposed, while not great, was large enough to prevent the segregation of subjects into one of the four exposure categories on the basis of a single breath sample collected more than $2 \mathrm{~h}$ after exposure. However, group-mean breathconcentration measurements did permit segregation of groups into the various exposure categories. The variation between subjects was due in part to errors in the breath sample collection technique, to leakage in $8-10 \%$ of the breath tubes, to analytical error, but also to genuine reproducible differences between individuals $(8,12)$.

The length of time after exposure in which the methylene chloride could be detected in the breath was related to the magnitude of exposure. The solvent was still detectable $16 \mathrm{~h}$ after exposure to 250 ppm for $7.5 \mathrm{~h}$, but was beneath detectable limits within a few hours after exposure to $50-100 \mathrm{ppm}$. Breath analysis in the first $2 \mathrm{~h}$ after exposure appeared to 
be the most reliable sampling time for estimating the time-weighted average vapor concentration for a $7.5-\mathrm{h}$ exposure to these lower concentrations.

The duration of exposure to a given vapor concentration of methylene chloride was an important variable influencing the rate of excretion of the solvent in the breath. This is shown in fig. 5. In this example, serial breath analyses following exposure to $500 \mathrm{ppm}$ would permit the construction of a decay curve which would indicate the probable duration of that exposure.

The female subjects had breath decay curves very similar to the male curves during the first hours after exposure (fig. 4). Their mean breath concentration $16 \mathrm{~h}$ after exposure to $250 \mathrm{ppm}$ was slightly higher than that recorded for similarly exposed male subjects.

The infrared analyses of the breath samples collected $15 \mathrm{~min}$ after exposure showed only abnormally high concentra- tions of $\mathrm{CO}$ in addition to the methylene chloride (12). The amount of $\mathrm{CO}$ present in the breath of nonsmokers was directly related to the magnitude of the exposure. The $\mathrm{CO}$ continued to increase for 1 to $2 \mathrm{~h}$ following the exposure and then decreased with a biological half-life twice as long as that which occurs following exogenous $\mathrm{CO}$ exposure (12).

\section{COMMENTS}

The analysis of expired breath for methylene chloride after exposure provides both an excellent diagnostic test and a measurement of the magnitude of exposure. The identification of the solvent in the breath constitutes an unequivocal diagnosis of exposure. Breath analysis at the appropriate time accurately reflects the body burden and the amount of the solvent absorbed. Thus it provides a noninvasive

Table $2 \mathrm{~g}$. Methylene chloride breath concentration of sedentary males exposed to $500 \mathrm{ppm}$.

\begin{tabular}{|c|c|c|c|c|c|c|c|}
\hline Time & Number & Mean & Range & Number & Mean & Range & $\mathrm{SD}$ \\
\hline & \multicolumn{3}{|c|}{ Single exposure for $1 \mathrm{~h}$} & \multicolumn{4}{|c|}{2 daily $1-\mathrm{h}$ exposures } \\
\hline $1 \mathrm{~min}$, preexit & 3 & 205.5 & $163.4-239.0$ & 6 & 212.8 & $163.4-242.2$ & 31.89 \\
\hline $1 \mathrm{~min}$, post & 3 & 119.7 & $82.7-178.2$ & 6 & 135.1 & $82.7-208$ & 50.17 \\
\hline 15 min, post & 3 & 59.8 & $31.4-90.5$ & 5 & 71.5 & $31.4-90.5$ & 26.35 \\
\hline $30 \mathrm{~min}$, post & 3 & 38.2 & $30.6-43.8$ & 6 & 39.5 & $22.5-53.5$ & 11.26 \\
\hline $60 \min$, post & 3 & 19.8 & $17.7-21.2$ & 5 & 21.7 & $17.7-26.8$ & 3.35 \\
\hline $120 \mathrm{~min}$, post & 3 & 4.5 & $3.4-5.8$ & 6 & 6.5 & $3.4-9.1$ & 2.38 \\
\hline $180 \mathrm{~min}$, post & 3 & 1.8 & $0.7-2.6$ & 5 & 2.6 & $0.7-4.2$ & 1.36 \\
\hline \multirow[t]{2}{*}{$1,350 \mathrm{~min}$, post } & 3 & 0.4 & $0.2-0.5$ & 6 & 0.4 & $0.2-0.5$ & 0.11 \\
\hline & \multicolumn{3}{|c|}{ Single exposure for $3 \mathrm{~h}$} & \multicolumn{4}{|c|}{2 daily $3-\mathrm{h}$ exposures } \\
\hline $1 \mathrm{~min}$, preexit & 3 & 218.3 & $182.0-260.0$ & 6 & 247.7 & $182.0-319.0$ & 46.69 \\
\hline $1 \mathrm{~min}$, post & 3 & 152.1 & $80.2-190.0$ & 6 & 193.0 & $80.2-254.8$ & 63.04 \\
\hline $15 \mathrm{~min}$, post & 3 & 109.0 & $98.1-116.0$ & 6 & 120.2 & $98.1-149.0$ & 19.38 \\
\hline $30 \mathrm{~min}$, post & 3 & 80.8 & $72.9-89.8$ & 6 & 88.1 & $72.9-108.0$ & 14.15 \\
\hline $60 \mathrm{~min}$, post & 3 & 44.5 & $41.7-48.4$ & 6 & 47.6 & $41.7-54.9$ & 6.02 \\
\hline $120 \mathrm{~min}$, post & 2 & 19.7 & $19.3-20.1$ & 4 & 16.9 & $12.0-20.1$ & 3.69 \\
\hline $180 \mathrm{~min}$, post & 3 & 2.4 & $1.0-4.3$ & 4 & 2.9 & $1.0-4.3$ & 1.64 \\
\hline \multirow[t]{2}{*}{$1,170 \mathrm{~min}$, post } & 3 & 0.5 & $0.3-0.7$ & 6 & 0.4 & $0.3-0.7$ & 0.14 \\
\hline & \multicolumn{3}{|c|}{ Single exposure for $7.5 \mathrm{~h}$} & \multicolumn{4}{|c|}{2 daily $7.5-\mathrm{h}$ exposures } \\
\hline $1 \mathrm{~min}$, preexit & 3 & 241.0 & $221.0-251.0$ & 7 & 276.8 & $221.0-333.0$ & 39.8 \\
\hline $1 \mathrm{~min}$, post & 3 & 187.7 & $158.0-215.0$ & 7 & 225.8 & $158.0-280.0$ & 42.77 \\
\hline $15 \mathrm{~min}$, post & 3 & 111.2 & $95.7-138.8$ & 7 & 146.8 & $95.7-201.0$ & 41.70 \\
\hline $30 \mathrm{~min}$, post & 3 & 89.1 & $82.6-98.1$ & 7 & 106.0 & $82.6-145.0$ & 26.15 \\
\hline $60 \mathrm{~min}$, post & 3 & 60.43 & $47.7-79.7$ & 7 & 66.2 & $47.7-94.5$ & 16.47 \\
\hline $120 \mathrm{~min}$, post & 3 & 24.9 & $24.0-25.3$ & 7 & 25.6 & $18.0-30.7$ & 4.20 \\
\hline $180 \min$, post & 3 & 15.1 & $6.0-22.0$ & 6 & 16.4 & $6.0-22.0$ & 5.41 \\
\hline $960 \mathrm{~min}$, post & 3 & 1.3 & $0.9-2.1$ & 6 & 1.4 & $0.9-2.1$ & 0.55 \\
\hline
\end{tabular}




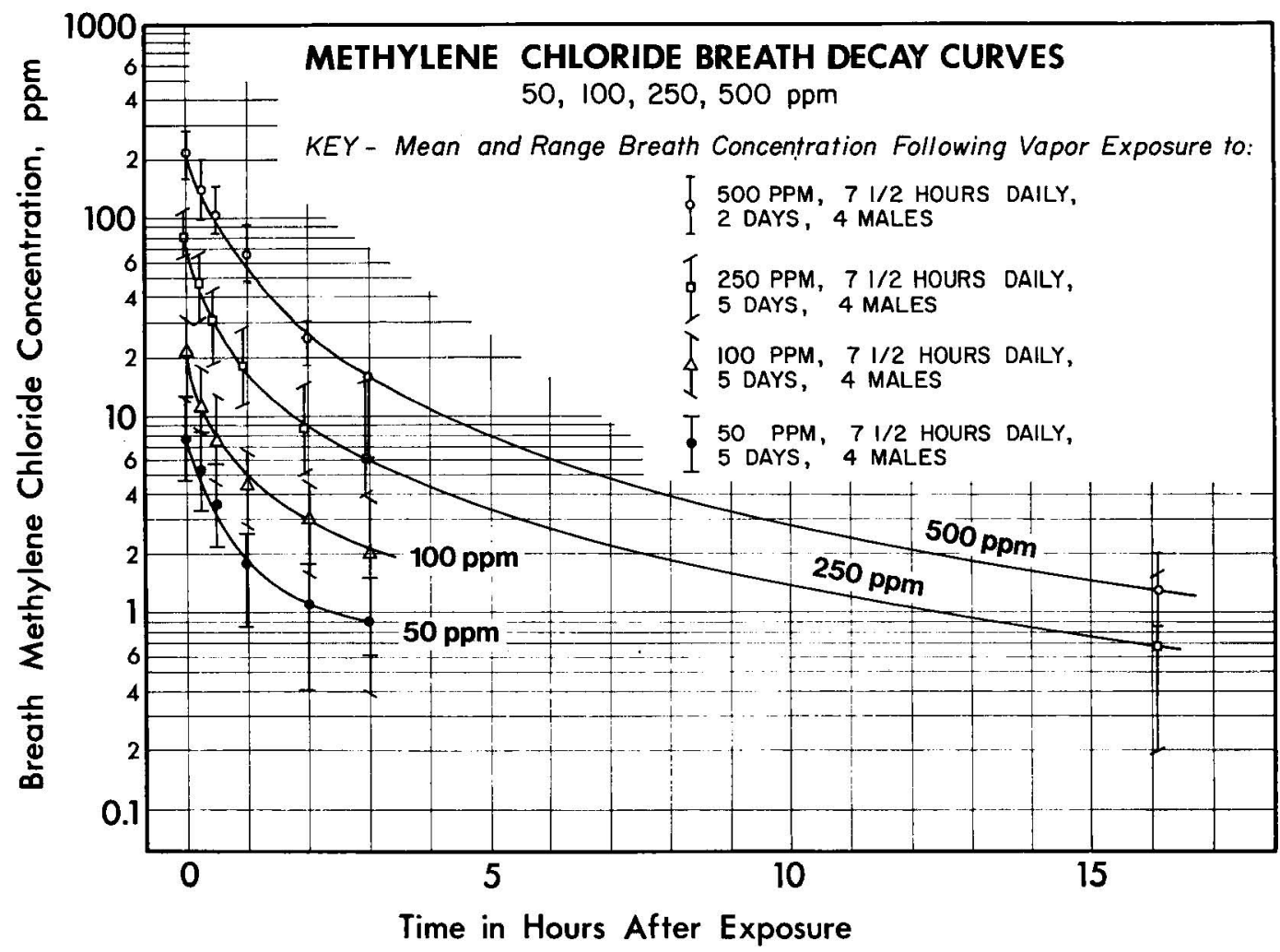

Fig. 3. Postexposure breath excretion curves for sedentary adults exposed repetitively for 7.5 h to $50,100,250$, or $500 \mathrm{ppm}$ of methylene chloride vapor.

method with which to monitor individual exposure to methylene chloride.

The analysis of the breath in the first $2 \mathrm{~h}$ after exposure provides a means for estimating the individualized exposure of the preceding $8 \mathrm{~h}$. Should the methylene chloride vapor concentration have been fluctuating widely toward the end of exposure, a breath sample obtained during the first hour following exposure may not indicate accurately the vapor concentration to which the subject had been exposed (13). A better time for the collection of a single breath sample with which to estimate the methylene chloride vapor exposure would be during the $1-$ to $2-\mathrm{h}$ postexposure interval when the solvent in the multiple tissue compartments is being released at a more uniform rate into the blood circulating to the lungs.

Experience in this laboratory indicates that it is feasible to allow the subject to collect breath samples in small glass tubes in the postexposure period when at home $(8,13)$. The breath samples should be collected in duplicate tubes to reduce the troublesome problem of leakage or breakage. A detailed discussion of the breath collection technique has been reported elsewhere $(8,13)$.

In contrast to 1,1,1-trichloroethane and tetrachloroethylene, which cumulate in the body with repetitive daily exposure, methylene chloride did not cumulate in the body sufficiently to affect the breath decay curves (table 2). Therefore, no correction factor need be considered when breath analysis is used to evaluate repetitive exposure.

Ideally, it would be desirable to construct individualized breath decay curves for each workman exposed to methylene chloride. This procedure would improve the predictability of the breath method by reducing variation due to true biological differences and breath collection tech- 


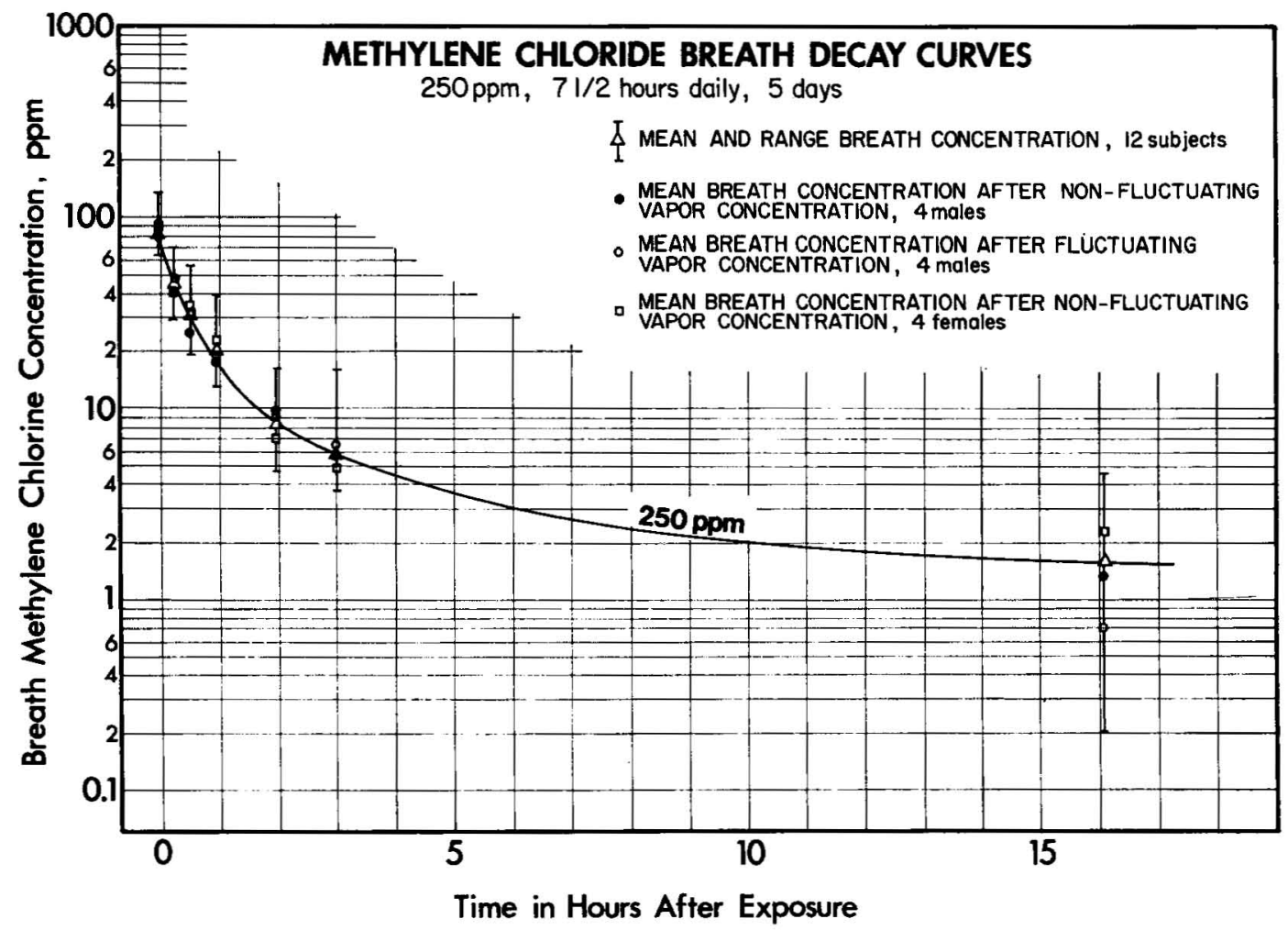

Fig. 4. Postexposure methylene chloride breath excretion curves for sedentary male and female subjects exposed daily for $7.5 \mathrm{~h}$ to $250 \mathrm{ppm}$.

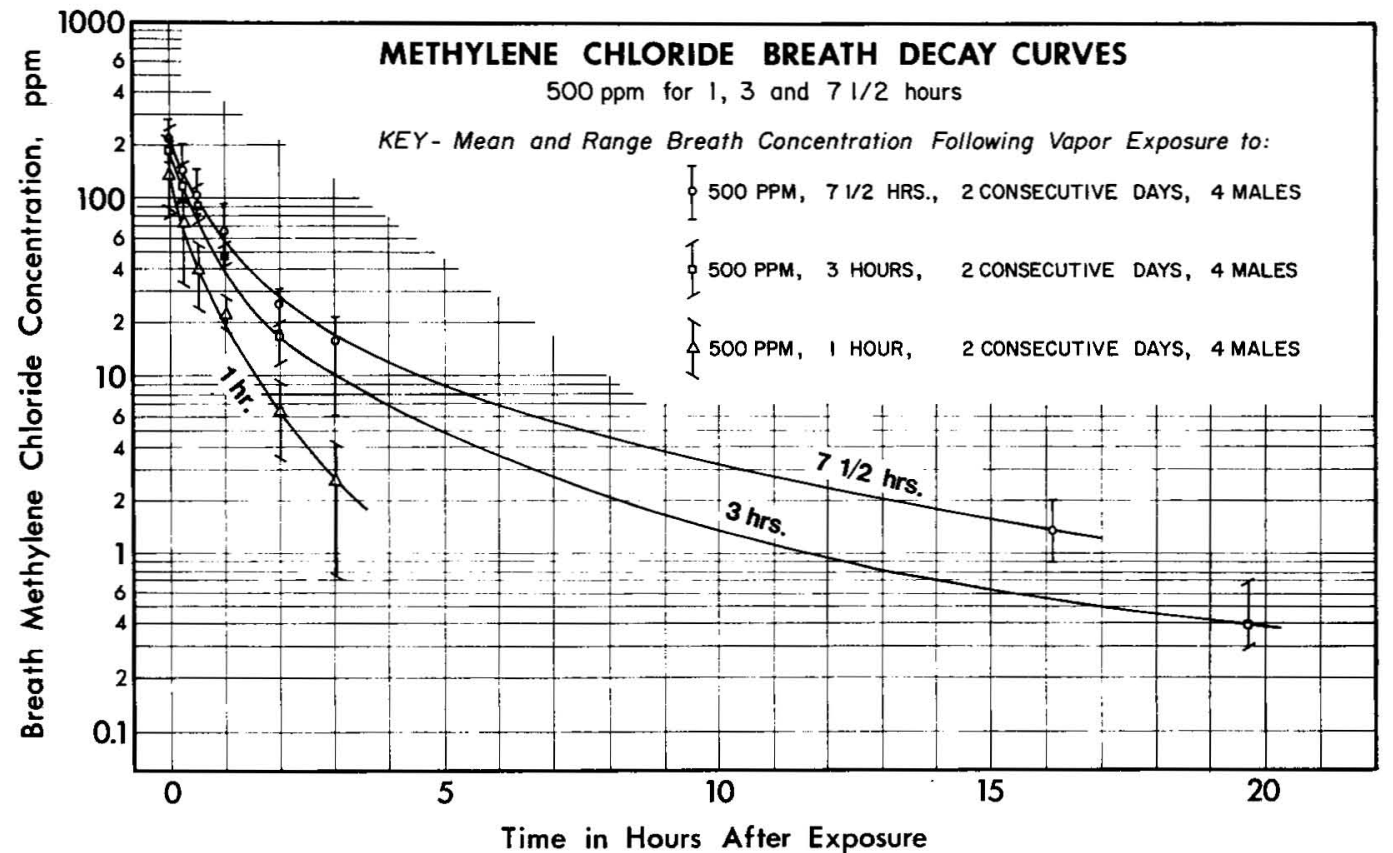

Fig. 5. Postexposure methylene chloride breath excretion curves for sedentary adults exposed repetitively to $500 \mathrm{ppm}$ for 1,3 , or $7.5 \mathrm{~h}$. 
niques. Generally this is not possible, but the breath data presented in table 2 and figs. $3-5$ should be adequate to determine whether overexposure has occurred.

The accuracy of the breath analysis technique in estimating methylene chloride exposure can be increased by collecting serial samples in the postexposure period. This procedure reduces the risk of error in extrapolating from a single data point, especially when that point occurs within the first hour following exposure. In the occupational setting it is often feasible to collect two breath samples. The first can be obtained when a workman leaves the exposure area, and followed by a second sampling 60 to $120 \mathrm{~min}$ later when he has arrived home. If these samples are collected in places free of heavy solvent contamination, background measurements need not be made $(8,13)$. The two or more breath values can then be compared with the breath decay curves most closely corresponding to the duration of exposure, and a judgment can be made as to whether overexposure had occurred.

It is important that the individual responsible for the interpretation of the breath data be cognizant of the fact that the data in table 2 were obtained from sedentary subjects. Doubling the minute respiratory volume during moderate exertion will increase the amount of methylene chloride absorbed and result in the excretion of significantly higher concentrations of the solvent in the expired breath. The toxicological effect will likewise be greater because the more active workman will absorb more solvent. Herein lies one of the greatest values of the breath method. It reflects the quantity of solvent absorbed and not merely the vapor concentration in the workplace.

For example, should one sedentary and one heavily exerting workman be exposed to an identical breathing zone concentration, $250 \mathrm{ppm}$ for $7.5 \mathrm{~h}$, the breath excretion curve for the sedentary person should fall within the range of values plotted for sedentary subjects in fig. 3 . The more active person with a higher minute respiratory volume would absorb more solvent during exposure and later excrete consistently higher amounts than shown in fig. 3. Thus the postexposure breath values will reflect more accurately the amount of solvent absorbed and the amount of $\mathrm{COHb}$ formed by each subject than could be estimated from breathing zone measurements alone. The postexposure breath data (table 2) can also be used to estimate the time-weighted average breathing-zone concentration, but only for sedentary subjects.

Because the biological half-life of the $\mathrm{COHb}$ derived from methylene chloride is twice that of the $\mathrm{COHb}$ derived from $\mathrm{CO}$, the use of serial breath analyses can be used to identify that portion of $\mathrm{COHb}$ arising from methylene chloride exposure from that formed as a result of exposure to exogenous CO $(6,15)$. For example, that portion of $\mathrm{COHb}$ due to the absorption $o^{f}$ $\mathrm{CO}$ in tobacco smoke will have a biological half-life of $5 \mathrm{~h}$ in contrast to the halflife of $10-12 \mathrm{~h}$ for $\mathrm{COHb}$ formed from methylene chloride metabolism. Serial breath analyses from a tobacco smoker exposed to methylene chloride will show a $\mathrm{COHb}$ excretion curve with a half-life intermediate between 5 and $10 \mathrm{~h}$. The greater the prolongation of the $\mathrm{COHb}$ halflife beyond $5 \mathrm{~h}$, the greater the $\mathrm{COHb}$ contribution from methylene chloride.

The reader should also be aware of the data demonstrating that the biological half-life of the $\mathrm{COHb}$ derived from methylene chloride can be further prolonged as the result of exposure to other substances, such as methyl alcohol (11), which triples the biological half-time.

The analysis of expired breath to measure methylene chloride absorption offers a practical noninvasive method for monitoring occupational exposure.

\section{ACKNOWLEDGMENTS}

This investigation was supported by Contract No. HSM-99-72-84 from the National Institute for Occupational Safety and Health.

Technical assistance was given by $\mathrm{H}$. V. Forster, Ph.D., A. J. Lebrun, M.D., J. H. Crespo, M.D., D. L. Crespo, M.D., S. A. Graff, K. K. Kujawski, P. E. Newton, M.S., D. L. Shekoski, R. J. Soto, M.S., and M. R. Stadler. 


\section{REFERENCES}

1. AMERICAN CONFERENCE OF GOVERNMENTAL INDUSTRIAL HYGIENISTS. Threshold limit values for substances in workroom air adopted by ACGIH for 1975. Cincinnati, Ohio 1975. $39 \mathrm{p}$.

2. ÁSTRAND, I., OVRUM, P. and CARLSSON, A. Exposure to methylene chloride: I. Its concentration in alveolar air and blood during rest and exercise and its metabolism. Scand. $j$. work environ. \& health 1 (1975) 78-94.

3. DIVINCENZO, G. D. and HAMILTON, M. L. Fate and disposition of methylene chloride in the rat. Toxicol. appl. pharmacol. 32 (1975) 385-393.

4. FODOR, G. G., PRAJSNAR, D. and SCHLIPKOTER, H. Endogenous CO formation by incorporated halogenated hydrocarbons of the methane series. Staub Reinhalt. Luft 33 (1973) 260-261.

5. KUBIC, V. L., ANDERS, M. W., ENGEL, R. R., BARLOW, C. H. and CAUGHEY, W. S. Metabolism of dihalomethanes to carbon monoxide. Drug metab. dispos. 2 " (1974) $53-57$.

6. PETERSON, J. E. Post-exposure relationship of carbon monoxide in blood and expired air. Arch. environ. health 21 (1970) $172-173$.

7. RATNEY, R S., WEGMAN, D. H. and ELKINS, H. B. In vivo conversion of methylene chloride to carbon monoxide. Arch. environ. health 28 (1974) 223-226.

8. STEWART, R. D. The use of breath analysis in clinical toxicology. In: W. HAYES (ed.), Essays in toxicology (vol. 5). Academic Press, New York, N.Y. 1974, chapt. 5.

9. STEWART, R. D., FISHER, T. N., HOSKO,
M. J., PETERSON, J. E., BARETTA, E. D. and DODD, H. C. Carboxyhemoglobin elevation after exposure to dichloromethane Science 176 (1972) 295-296.

10. STEWART, R. D., FISHER, T. N., HOSKO, M. J., PETERSON, J. E., BARETTA, E. D. and DODD, H. C. Experimental human exposure to methylene chloride. Arch. environ. health 25 (1972) $324-348$.

11. STEWART, R. D. and HAKE, C. L. Paintremover hazard. $J$. am. med. assoc. 235 (1976) $398-401$.

12. STEWART, R. D., HAKE, C. L., FORSTER, H. V., LEBRON, A. J., PETERSON, J. E. and WU., A. Methylene chloride: Development of a biologic standard for the industrial worker by breath analysis (Report no. NIOSH-MCOW-ENVM-MC-74-9). National Institute of Occupational Safety and Health, Cincinnati, Ohio 1974.

13. STEWART, R. D., HAKE, C. L. and PETERSON, J. E. Use of breath analysis to monitor trichloroethylene exposures. Arch. environ. health 29 (1974) 6-13.

14. STEWART, R. D., PETERSON, J. E., HAKE, C. L., LEBRUN, A. J. and NEWTON, P. E. Measurement of physiological and behavioral responses in a controlledenvironment chamber. In: C. XINTARAS, B. L. JOHNSON and I. DE GROOT (eds.), Behavioral toxicology: Early detection of occupational hazards. (HEW publication no. (NIOSH) 74-126), National Institute for Occupational Safety and Health, Washington, D. C. 1974 , pp. $361-375$.

15. STEWART, R. D., STEWART, R. S., STAMM, W. and SEELEN, R. P. Rapid estimation of carboxyhemoglobin level in fire fighters. $J$. am. med. assoc. 235 (1976) $390-392$. 